\title{
Association of Lupus Anticoagulant with Brain Atrophy in Gulf War Illness (GWI)
}

\author{
Lisa M. James ${ }^{1,2,3}$, Peka Christova ${ }^{1,2}$, Rachel A. Johnson' ${ }^{1}$, Brian E. Engdahl'1,2,4, Scott M. Lewis ${ }^{1,5}$, Adam F. Carpenter ${ }^{1,5}$, \\ Apostolos P. Georgopoulos $1,2,3,5 *$ \\ 'Brain Sciences Center, Department of Veterans Affairs Health Care System, Minneapolis, MN, 55417, USA \\ Department of Neuroscience, University of Minnesota Medical School, Minneapolis, MN 55455, USA \\ ${ }^{3}$ Department of Psychiatry, University of Minnesota Medical School, Minneapolis, MN 55455, USA \\ ${ }^{4}$ Department of Psychology, University of Minnesota Medical School, Minneapolis, MN 55455, USA \\ ${ }^{5}$ Department of Neurology, University of Minnesota Medical School, Minneapolis, MN 55455, USA
}

Article Info

\section{Article Notes}

Received: April 23, 2021

Accepted: May 25, 2021

\section{${ }^{*}$ Correspondence:}

*Apostolos P. Georgopoulos, Brain Sciences Center (11B), Minneapolis VAHCS, One Veterans Drive, Minneapolis, MN 55417, USA; Email: omega@umn.edu.

(C) 2021 Georgopoulos AP. This article is distributed under the terms of the Creative Commons Attribution 4.0 International License.

\section{Keywords}

Lupus anticoagulant

Brain volume

Gulf War Illness

Antiphospholipid antibodies

Autoimmunity

\section{ABSTRACT}

Separate lines of research have documented brain atrophy and evidence of autoimmune mechanisms in Gulf War Illness (GWI), including the presence of lupus anticoagulant (LAC), in veterans with GWI. Here we evaluated the possible association of LAC and brain volume in veterans with GWI. The presence of LAC was determined using Silica Clotting Time and dilute Russell's Viper Venom Time assays. MRI data was acquired using a Philips 3T MR scanner from which total gray matter, total cortical gray matter, total subcortical gray matter, and total cerebral white matter were derived. The results demonstrated a statistically significant reduction of brain volume in all regions tested in GWI veterans with positive LAC, as compared to those without LAC. These findings add to the literature implicating autoimmune mechanisms in $\mathrm{GWI}$ and point to the presence of prothrombotic antiphospholipid antibodies as contributing to brain atrophy in GWI.

\section{Introduction}

One-third of U.S. Veterans of the 1990-91 Persian Gulf War are affected by Gulf War Illness (GWI), a condition characterized by chronic and often disabling symptoms involving multiple organ systems including, prominently, the brain $^{1,2}$. Numerous brain abnormalities have been associated with $\mathrm{GWI}^{3-9}$. For instance, several recent studies have documented brain atrophy in veterans with $\mathrm{GWI}^{7-11}$. In addition, brain functional anomalies in veterans with GWI have been shown to be indistinguishable from those of known autoimmune disorders ${ }^{6}$, supporting theories that GWI may, in part, be related to autoimmunity ${ }^{12-14}$. Providing further support for autoimmune processes in GWI, we recently reported lupus anticoagulant (LAC) positivity in one-quarter of veterans with GWI, a rate that was comparable to that found in veterans diagnosed with well-established autoimmune disorders ${ }^{15}$. LAC, an indicator of autoimmunity, is an autoantibody against phospholipids or phospholipid-binding proteins that results in formation of blood clots in vivo. LAC has been associated with ischemic brain changes including brain atrophy in systemic lupus erythematosus (SLE) ${ }^{16}$ and neuropsychiatric $\operatorname{SLE}^{17}$. Here we evaluated the association of LAC and brain volume in veterans with GWI.

\section{Methods}

Sixty-eight veterans (64 men) who met criteria for GWI and no diagnosed autoimmune conditions participated in this study as paid volunteers (see $\mathrm{ref}^{8}$ for GWI diagnostic criteria). This sample 
consisting of veterans studied using magnetic resonance imaging (MRI) is a subset of a larger one of 84 veterans studied for Lupus Anticoagulant (LAC) positivity ${ }^{15}$. The Minneapolis VA Health Care System (VAHCS) Pathology and Laboratory Medicine Service evaluated blood samples for markers related to inflammation and autoimmunity including C-reactive protein (CRP), C3, C4, platelet count, and LAC. LAC was assessed using a standard Lupus Inhibitor Panel (VA Document ID: HEM02-023, issue date: 11/4/2019) consisting of Silica Clotting Time (SCT) and dilute Russell's Viper Venom time (dRVVT), with both screen and confirm tests being run simultaneously for both tests. Cutoff values were 1.19 for SCT C test ratio and 1.15 for dRVVT $C$ test ratio; the Lupus Inhibitor Panel outcome was Negative if both values were below cutoffs and Positive if either value was above the cutoff. MRI data was acquired using a Philips 3T MR scanner (as detailed in $\mathrm{ref}^{8}$ ) from which we derived the volumes of the following regions using Freesurfer: total gray matter, total cortical gray matter, total subcortical gray matter, and total cerebral white matter. The study was approved by the University of Minnesota and Minneapolis VA Health Care System Institutional Review Boards. Standard statistical methods including descriptive statistics (mean, SEM, etc.) and analysis of covariance (ANCOVA) were used to analyze the data in IBM-SPSS (version 26). Separate ANCOVAs were performed for each brain measure.

\section{Results}

The age $($ mean \pm SEM) was $56.39 \pm 1.15$ for men $(\mathrm{N}=$ 64) and $48.87 \pm 2.5$ for women $(\mathrm{N}=4)$; these means did not differ significantly $\left(\mathrm{t}_{[66]}=1.61, \mathrm{P}=0.11\right)$. LAC was present in 20/68 (29.4\%; all men) participants in this sample. This percentage is similar to those found in veterans with relapsing remitting multiple sclerosis (33.3\%), rheumatoid arthritis (33.3\%), Sjögren's syndrome $(12.5 \%)$ or lupus $(28.6 \%)^{15}$. Age did not differ significantly between the LAC- and LAC+ groups (mean \pm SEM: LAC-absent, 56.41 $\pm 1.39, \mathrm{~N}=40$; LA-present, $54.82 \pm 1.76, \mathrm{~N}=28 ; \mathrm{t}_{[66]}=$ $0.652, \mathrm{P}=0.517$ ). A multivariate ANCOVA was used to

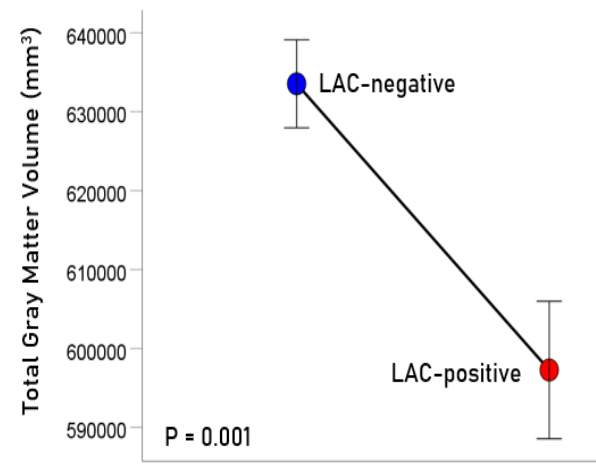

Figure 1. Mean ( \pm SEM) of total gray matter volume for the LACnegative and LAC-positive groups. Means are adjusted for total intracranial volume, age and gender (ANCOVA).

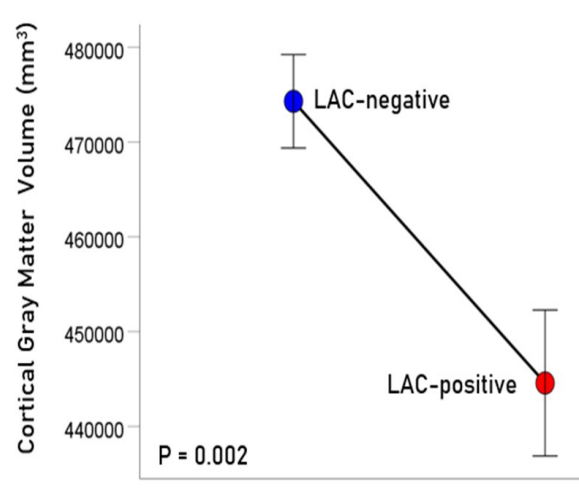

Figure 2. Mean ( \pm SEM) of cortical gray matter volume for the LACnegative and LAC-positive groups. Means are adjusted for total intracranial volume, age and gender (ANCOVA).

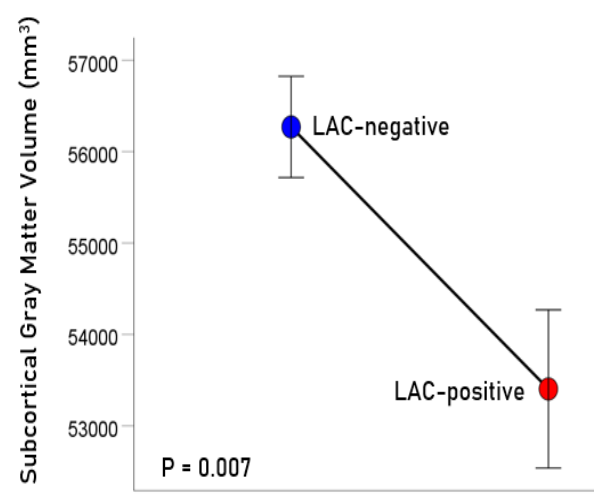

Figure 3. Mean ( \pm SEM) of subcortical gray matter volume for the LAC-negative and LAC-positive groups. Means are adjusted for total intracranial volume, age and gender (ANCOVA).

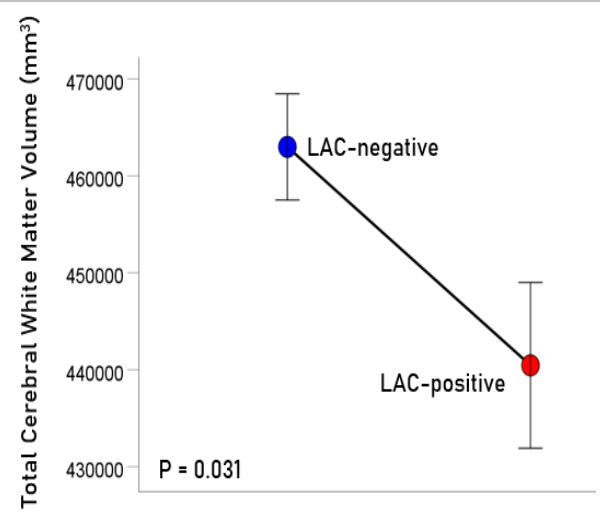

Figure 4. Mean ( \pm SEM) of total cerebral white matter volume for the LAC-negative and LAC-positive groups. Means are adjusted for total intracranial volume, age and gender (ANCOVA).

evaluate the association of LAC and each brain volume measure, with total intracranial volume, age, and gender as covariates. (An initial analysis showed that CRP, C3, $\mathrm{C} 4$, and platelet count did not have a significant effect on any of the measures of brain volume and were not used in subsequent tests.) There was a statistically significant reduction of brain volume in the $\mathrm{LAC}+$ group in all regions tested (Figures 1-4), by an average of 5.7\% (Table 1). 
Table 1: Statistics for measures and comparisons between LAC groups. Means are adjusted for age, gender and total intracranial volume.

\begin{tabular}{|c|c|c|c|c|c|c|}
\hline \multirow{2}{*}{ Region } & \multicolumn{2}{|c|}{ LAC negative $(\mathrm{N}=40)$} & \multicolumn{2}{|c|}{ LAC positive ( $N=28)$} & \multicolumn{2}{|c|}{ Group comparisons (LAC positive) - LAC negative) } \\
\hline & Mean $\left(\mathrm{mm}^{3}\right)$ & SEM & Mean $\left(\mathrm{mm}^{3}\right)$ & SEM & \% change & P-value (F-test, ANCOVA) \\
\hline Total gray matter & 633541 & 5574 & 597276 & 8708 & $-5.72 \%$ & 0.001 \\
\hline Cortical gray matter & 474296 & 4929 & 444581 & 7700 & $-6.26 \%$ & 0.002 \\
\hline Subcortical gray matter & 56271 & 554 & 53404 & 866 & $-5.10 \%$ & 0.007 \\
\hline Cerebral white matter & 462993 & 5474 & 440461 & 8552 & $-4.87 \%$ & 0.031 \\
\hline
\end{tabular}

\section{Discussion}

Here we evaluated the association of lupus anticoagulant and brain volume in veterans with GWI and found that brain volume was significantly reduced in GWI veterans with positive lupus anticoagulant compared to those without lupus anticoagulant. These findings add to the literature implicating autoimmune mechanisms in GWI and suggest a potential role for prothrombotic antiphospholipid autoantibodies in GWI-related brain atrophy.

To our knowledge, this is the first study to link brain atrophy in GWI to the presence of antiphospholipid antibodies; however, antiphospholipid antibodies have been associated with several brain abnormalities in other conditions. LAC, in particular, has been associated with evidence of small vessel disease including white matter hyperintensities, microhemorrhage, and cortical atrophy in patients with neuropsychiatric SLE ${ }^{17}$. Similarly, LAC, but not other antiphospholipid antibodies, has been found to be associated with white matter lacunar infarcts and atrophy in patients with SLE ${ }^{16}$. In addition, LAC has been associated with cognitive dysfunction ${ }^{18}$ in patients with SLE and with neuropsychiatric manifestations ${ }^{19}$ of SLE. Likewise, antiphospholipid syndrome, which is defined in part by the presence of antiphospholipid antibodies including LAC, has also been widely associated with similar neuroimaging abnormalities including infarcts, white matter hyperintensities, atrophy, and microstructural white matter abnormalities ${ }^{20}$ as well as cognitive dysfunction ${ }^{21}$. Although the mechanisms of brain involvement in antiphsopholipid syndrome and SLE are not well understood, evidence supports a role of vasculopathy ${ }^{22-24}$, thrombosis ${ }^{25-26}$, and/or direct binding of antiphospholipid antibodies to neurons and glia $^{20}$. The mechanisms underlying the association of LAC and brain atrophy in veterans with GWI remain to be elucidated. Furthermore, the cause of LAC positivity in GWI veterans is unclear although antiphospholipid antibodies typically occur as a result of molecular mimicry between phospholipid proteins and those of foreign antigens (e.g., infections, vaccines) $)^{27-29}$.

The present findings shed new light on brain abnormalities in GWI and support a role of autoimmune mechanisms in GWI-related atrophy. The findings, however, must be considered within the context of study limitations including a relatively small sample of predominantly male
GWI veterans, absence of a control group, a cross-sectional study design, and evaluation of antiphospholipid antibody concentrations at a single time point. Longitudinal studies in larger samples of Gulf War veterans will be beneficial for evaluating the long-term impact of LAC-positivity on brain structure and function in veterans with and without GWI.

\section{Conclusion}

This study documents autoimmune-associated brain atrophy in veterans with GWI, providing further evidence of GWI as a neuroimmune condition ${ }^{6}$. In addition to the brain effects observed here and elsewhere ${ }^{16-21}$, LAC is a risk factor for prothrombotic events ${ }^{30}$ and myocardial infarction ${ }^{31}$, both of which occur at significantly higher rates in male Gulf War veterans compared to the general population ${ }^{32}$. Thus, it may be prudent to monitor Gulf War veterans for evidence of hypercoagulation to reduce further deleterious health effects associated with LAC-positivity.

\section{Acknowledgements}

This work was partially supported by the Department of Defense (Award Number W81XWH-15-1-0520), University of Minnesota (the American Legion Brain Sciences Chair) and the U.S. Department of Veterans Affairs. The sponsors had no role in the current study design, analysis or interpretation, or in the writing of this paper. The contents do not represent the views of the U.S. Department of Veterans Affairs or the United States Government.

\section{Author Contributions}

Contributed to data collection: LMJ, AFC, SML, RAJ. Contributed to participant recruitment and evaluation: BEE, LMJ, RAJ. Contributed to study design: APG, BEE, LMJ, AFC. Contributed to data analysis: APG, PC. Wrote the paper: LMJ, APG. Contributed to editing the paper: All.

\section{Conflicts of Interest}

None.

\section{References}

1. Fukuda K, Nisenbaum R, Stewart G, et al. Chronic multisymptom illness affecting Air Force veterans of the Gulf War. JAMA. 1998; 280(11): 981-988.

2. Steele L. Prevalence and patterns of Gulf War illness in Kansas veterans: association of symptoms with characteristics of person, place, and time of military service Am J Epidemiol. 2000; 152: 9921002.

3. White RF, Steele L, O'Callaghan JP, et al. Recent research on Gulf War 
illness and other health problems in veterans of the 1991 Gulf War: Effects of toxicant exposures during deployment. Cortex. 2016; 74: 449-75.

4. Engdahl BE, James LM, Miller RD, et al. Brain function in Gulf War Illness (GWI) and associated mental health comorbidities. J Neurol Neuromedicine. 2018; 3(4): 24-34.

5. Engdahl BE, James LM, Miller RD, et al. A magnetoencephalographic (MEG) study of Gulf War Illness (GWI). EBioMedicine. 2016; 12: 12732.

6. Georgopoulos AP, James LM, Carpenter AF, et al. Gulf War illness (GWI) as a neuroimmune disease. Exp Brain Res. 2017; 235(10): 3217-3225.

7. Christova P, James LM, Engdahl BE, et al. Subcortical brain atrophy in Gulf War Illness. Exp Brain Res. 2017; 235: 2777-2786.

8. Christova P, James LM, Carpenter AF, et al. Gulf War Illness: C-reactive protein is associated with reduction of the volume of hippocampus and decreased fractional anisotropy of the fornix. J Neurol Neuromedicine. 2020; 5(3): 6-15.

9. Christova P, James LM, Carpenter AF, et al. C-Reactive Protein is Associated with Brain White Matter Anomalies in Gulf War Illness. J Neurol Neuromedicine. 2020; 5(3): 55-62.

10. Rayhan RU, Stevens BW, Raksit MP, et al. Exercise challenge in Gulf War Illness reveals two subgroups with altered brain structure and function. PLoS One. 2013; 8(6): e63903.

11. Zhang Y, Avery T, Vakhtin AA, et al. Brainstem atrophy in Gulf War illness. NeuroToxicology. 2020; 78: 71-9.

12. Israeli E. Gulf War syndrome as a part of the autoimmune (autoinflammatory) syndrome induced by adjuvant (ASIA). Lupus. 2012; 21(2): 190-4.

13. Moss JI. Gulf War illnesses are autoimmune illnesses caused by increased activity of the p38/MAPK pathway in CD4+ immune system cells, which was caused by nerve agent prophylaxis and adrenergic load. Med Hypotheses. 2013; 81(6): 1002-3.

14. Shoenfeld Y, Agmon-Levin N. 'ASIA'-autoimmune/inflammatory syndrome induced by adjuvants. J Autoimmun. 2011; 36(1): 4-8.

15. James LM, Johnson RA, Lewis SM, et al. Lupus Anticoagulant in Gulf War Illness and Autoimmune Disorders: A Common Pathway Toward Autoimmunity. J Immunological Sci. 2021; 5(1): 14-18.

16. Magro-Checa C, Kumar S, Ramiro S, et al. Are serum autoantibodies associated with brain changes in systemic lupus erythematosus? MRI data from the Leiden NP-SLE cohort. Lupus. 2019; 28(1): 94-103.

17. Sarbu N, Alobeidi F, Toledano P, et al. Brain abnormalities in newly diagnosed neuropsychiatric lupus: Systematic MRI approach and correlation with clinical and laboratory data in a large multicenter cohort. Autoimmun Rev. 2015; 14: 153-159.
18. Denburg SD, Denburg JA. Cognitive dysfunction and antiphospholipid antibodies in systemic lupus erythematosus. Lupus. 2003; 12(12): 883-90.

19. Zambrano YC, Ramos JD, Vargas NE, et al. Risk factors for neuropsychiatric manifestations in children with systemic lupus erythematosus: case-control study. Pediatr Neurol. 2014; 51(3): 4039.

20. Ricarte IF, Dutra LA, Abrantes FF, et al. Neurologic manifestations of antiphospholipid syndrome. Lupus. 2018; 27(9): 1404-14.

21. Tektonidou MG, Varsou N, Kotoulas G, et al. Cognitive deficits in patients with antiphospholipid syndrome: association with clinical, laboratory, and brain magnetic resonance imaging findings. Arch Int Med. 2006; 166(20): 2278-84.

22. Ramos-Casals M, Nardi N, Lagrutta M, et al. Vasculitis in systemic lupus erythematosus: prevalence and clinical characteristics in 670 patients. Medicine. 2006; 85(2): 95-104.

23. Cieślik P, Hrycek A \& Kłuciński P. (2008). Vasculopathy and vasculitis in systemic lupus erythematosus. Pol Arch Med Wewn. 2008; 118(12): 57-63.

24. Lally L, Sammaritano LR. Vasculitis in antiphospholipid syndrome. Rheum Dis Clin N AM. 2015; ;41(1): 109-23.

25. Greaves M. Antiphospholipid antibodies and thrombosis. The Lancet. 1999; 353(9161): 1348-53.

26. Love PE, Santoro SA. Antiphospholipid antibodies: anticardiolipin and the lupus anticoagulant in systemic lupus erythematosus (SLE) and in non-SLE disorders: prevalence and clinical significance. Ann Intern Med. 1990; 112(9): 682-98.

27. Asherson RA, Cervera R. Antiphospholipid antibodies and infections. Ann Rheum Dis. 2003; 62(5): 388-93.

28. Cruz-Tapias P, Blank M, Anaya JM, et al. Infections and vaccines in the etiology of antiphospholipid syndrome. Curr Opin Rheumatol. 2012; 24(4): 389-93.

29. Reyes Gil M, Barouqa M, Szymanski J, et al. Assessment of lupus anticoagulant positivity in patients with coronavirus disease 2019 (COVID-19). JAMA Netw Open. 2020; 3(8): e2017539.

30. Galli M, Luciani D, Bertolini G, et al. Lupus anticoagulants are stronger risk factors for thrombosis than anticardiolipin antibodies in the antiphospholipid syndrome: a systematic review of the literature. Blood. 2003; 101: 1827-32.

31. Petri M. Update on anti-phospholipid antibodies in SLE: The Hopkins' Lupus Cohort. Lupus. 2010; 19(4): 419-423.

32. Zundel CG, Krengel MH, Heeren T, et al. Rates of chronic medical conditions in 1991 Gulf War veterans compared to the general population. Int J Environ Res Pub He. 2019; 16(6): 949. 